\section{Gut microbiome composition and risk factors in a large cross-sectional IBS cohort}

To cite: Agnello M, Carroll LN, Imam N, et al. Gut microbiome composition and risk factors in a large cross-sectional IBS cohort. BMJ Open Gastro 2020;7:e000345. doi:10.1136/ bmjgast-2019-000345

- Additional material is published online only. To view please visit the journal online (http://dx.doi.org/10.1136/ bmjgast-2019-000345).

MA and LNC contributed equally.

Received 5 October 2019 Revised 30 December 2019 Accepted 9 January 2020

Check for updates

C Author(s) (or their employer(s)) 2020. Re-use permitted under CC BY-NC. No commercial re-use. See rights and permissions. Published by BMJ.

${ }^{1}$ Medical Affairs, uBiome, San Francisco, California, USA ${ }^{2}$ Medical Affairs, uBiome, Santiago, Chile

${ }^{3}$ Bioinformatics, uBiome, Santiago, Chile

${ }^{4}$ Data Science, uBiome, Santiago, Chile

Correspondence to Dr Michael C Hoaglin; mike@hoaglin.com

\section{ABSTRACT}

Objective Irritable bowel syndrome (IBS) is a common gastrointestinal disorder that is difficult to diagnose and treat due to its inherent heterogeneity and unclear aetiology. Although there is evidence suggesting the importance of the microbiome in IBS, this association remains poorly defined. In the current study, we aimed to characterise a large cross-sectional cohort of patients with self-reported IBS in terms of microbiome composition, demographics, and risk factors.

Design Individuals who had previously submitted a stool sample for $16 \mathrm{~S}$ microbiome sequencing were sent a comprehensive survey regarding IBS diagnosis, demographics, health history, comorbidities, family history, and symptoms. Log ratio-transformed abundances of microbial taxa were compared between individuals reporting a diagnosis of IBS without any comorbidities and individuals reporting no health conditions. Univariable testing was followed by a multivariable logistic regression model controlling for relevant confounders.

Results Out of 6386 respondents, 1692 reported a diagnosis of IBS without comorbidities and 1124 reported no health conditions. We identified 3 phyla, 15 genera, and 19 species as significantly associated with IBS after adjustment for confounding factors. Demographic risk factors include a family history of gut disorders and reported use of antibiotics in the last year.

Conclusion The results of this study confirm important IBS risk factors in a large cohort and support a connection for microbiome compositional changes in IBS pathogenesis. The results also suggest clinical relevance in monitoring and investigating the microbiome in patients with IBS. Further, the exploratory models described here provide a foundation for future studies.

\section{INTRODUCTION}

Clinical research from the last 20 years has dramatically advanced the understanding and classification of chronic gastrointestinal (GI) conditions. ${ }^{12}$ Despite great strides towards clarifying the pathophysiology of some GI conditions, irritable bowel syndrome (IBS) remains a challenge to define and diagnose. ${ }^{34}$ Current diagnostic work-up involves ruling out other conditions and meeting

\section{Summary box}

What is already known about this subject?

- Irritable bowel syndrome (IBS) is poorly defined and difficult to diagnose due to the heterogeneous nature of the disease.

- The gut microbiome supports essential health functions, and alterations in the microbiome are associated with a variety of gut and metabolic conditions including IBS.

What are the new findings?

- Large sample size allows for detailed comparison of microbial and demographic risk factors between a population reporting a diagnosis of IBS and a control group reporting no health conditions.

- Novel approach leveraging the compositional nature of microbiome uses species-level pairwise ratios to highlight relationships between species.

- Pathogenic species may alter the microbiome environment to increase or decrease the risk of IBS; study findings support a role for microbial dysbiosis in IBS pathogenesis.

How might it impact on clinical practice in the foreseeable future?

- Microbiome testing to further define dysbiosis among patients with IBS supports improved classification of a loosely defined disease, and such testing may impact screening guidelines for chronic gastrointestinal symptoms as well as help reduce morbidity and healthcare costs.

clinical criteria, such as the recently updated Rome IV criteria. ${ }^{4}$ The loose collection of symptoms related to abdominal pain and changes in stool frequency or appearance can be difficult to separate from other GI conditions, further complicating the diagnostic process. ${ }^{3-5}$ Patients often receive repeated tests and procedures across multiple providers in order to resolve their symptoms. ${ }^{67}$ Patients with IBS also often rank low on quality of life scores and have high rates of anxiety and depression. ${ }^{28}$ Treatment for IBS 
is multifaceted, typically targeting individual symptom reduction through the use of antidepressants, antibiotics, motility agents, diet changes, and others. ${ }^{3}{ }^{10}$

Still, IBS is one of the most commonly diagnosed GI conditions, affecting over $11 \%$ of the global population, ${ }^{8}$ reflecting a great need to better understand the disease and its mechanisms. Research has demonstrated that IBS may be ascribed to a dynamic, personalised mix of internal and external conditions, including host genetics and immune response, environment, stress, diet, and gut microbiome. ${ }^{811}$ Additional risk factors include broadspectrum antibiotic exposure ${ }^{12}$ and GI infection (eg, gastroenteritis). ${ }^{13}$ Current research focuses on identifying microbial signatures for IBS to aid in diagnosis and treatment. $^{1214}$

The last several years have seen a critical paradigm shift in the approach towards characterising the gut microbiome of patients with IBS. Scientific and clinical research responding to advances in clinical microbiology has expanded beyond the 'one microbe-one disease' paradigm to explore complex diseases such as IBS, and studies are embracing a more ecological perspective that may be better suited to identify microbial signatures signalling dysbiosis. ${ }^{11}$ Ongoing efforts suggest that there are distinct, detectable microbial differences in patients with IBS compared with healthy individuals, ${ }^{10} 1214$ and studies exploring microbiome dysbiosis suggest that an altered microbiome may make an individual more susceptible to disease. ${ }^{11}{ }^{15-18}$ However, such efforts for IBS have been hampered by the multifactorial nature of IBS, lack of a standard test, varied patient populations and methodologies, and small sample sizes. ${ }^{214}{ }^{19-21}$ Additional research is needed to investigate relationships between micro-organisms of the gut to help provide insights into disease state conditions and to support clinicians as they endeavour to treat IBS.

This study uses gut microbiome and survey data from a cohort of over 1500 individuals reporting a diagnosis of IBS and compares the findings with an equivalently sized cohort reporting no health issues in order to more fully characterise the gut microbiome in IBS. Importantly, this exploratory study leverages the compositional nature of the gut microbiome through species-level analyses and pairwise ORs. Using these methods to investigate the microbiome in a large cohort reporting an IBS diagnosis will help to elucidate microbial patterns for IBS and adds to the growing evidence for microbiome testing utility in the diagnostic process of undifferentiated chronic GI symptoms.

\section{METHODS}

\section{Participants and data collection}

All users of a commercial gut microbiome testing kit (uBiome, San Francisco, California) residing in the USA were given the option of participating in this study, which included sequencing of the microbiome from a stool sample and collection of demographic and health data via questionnaires. In May 2018, all consented individuals who sent in a stool sample and received results between January 2017 and May 2018 ( $\mathrm{n}=28878)$ were sent a survey via email. The survey was open for 1 month. The survey assessed IBS status in two ways: whether the person thought they had IBS at the time of their most recent sampling and whether a healthcare provider had ever diagnosed them with a list of 25 various health conditions (including IBS). The survey also gathered information on symptoms, treatment, outcomes, health history, family history, and healthcare system utilisation. The Strengthening the Reporting of Observational Studies in Epidemiology guidelines were used to ensure proper reporting of this observational study. ${ }^{22}$ Due to the large sample size, study participants were not involved in the research design or discussion of results.

\section{Microbiome sequencing and analysis}

Stool samples were collected at home by individuals per the testing kit instructions. Participants were instructed to use the sterile swab included in the kit to transfer a small amount of faecal material into the sample collection vial, which contains a lysis and stabilisation buffer. Samples were then mailed to the laboratory for analysis. For DNA extraction, samples were first lysed via bead-beating, and DNA was extracted in a class 1000 cleanroom using a liquid-handling robot by a guanidine thiocyanate silica column-based purification method. ${ }^{23}{ }^{24}$ The V4 variable region of the 16S rRNA gene was amplified via PCR with universal primers (515F: GTGCCAGCMGCCGCGGTAA and 806R: GGACTACHVGGGTWTCTAAT). ${ }^{25}$ Indexed PCR products were pooled, column-purified, and sizeselected through microfluidic DNA fractionation. ${ }^{26}$ Consolidated libraries were quantified by quantitative PCR using the Kapa Bio-Rad iCycler qPCR kit on a Bio$\mathrm{Rad} \mathrm{MyiQ}$ and sequenced on the Illumina NextSeq 500 platform rendering $2 \times 150 \mathrm{bp}$ paired-end sequences.

After sequencing, BCL2FASTQ software was used to demultiplex the data and generate individual fastq files per sample. Reads with Q-score $<30$ were excluded from the analysis. Primers were removed and paired forward and reverse reads were appended together. Resulting amplicons were clustered using the Swarm V.2.1.5 algorithm with a distance of one nucleotide and the 'fastidious' and 'usearch-abundance' flags. ${ }^{27}$ The most abundant sequence per cluster was considered the real biological sequence and was assigned the count of all reads in the cluster. Chimera sequences were removed using the VSEARCH algorithm..$^{28}$ Reads passing all the above filters were aligned using $99 \%$ identity over $100 \%$ of the length against a hand-curated database of target $16 \mathrm{~S}$ rRNA gene sequences and taxonomic annotations derived from the SILVA V.132 database. ${ }^{29}$ Only taxa for which V4 amplicons allow unequivocal assignment of taxonomic membership were included in this study. Raw counts were transformed using a centred log ratio (CLR) for analysis. ${ }^{30}$ 


\section{Statistical analyses}

IBS cases were identified as those participants who answered 'yes' to the question 'To the best of your knowledge, did you have the diagnosis of irritable bowel syndrome (IBS) at the time of your most recent stool sample?' Only individuals reporting an IBS diagnosis and no comorbidities (eg, cardiovascular disease, cancer, kidney disease) were included. A control group was generated from participants who responded 'no' to the aforementioned question and reported no other comorbidities or conditions. Less than $0.1 \%$ of the data were missing and no imputation was performed.

Descriptive statistics for participant characteristics were calculated and compared across IBS and control groups. Wilcoxon rank-sum test was used to compare age distributions in the IBS and control groups. Exact proportion tests were performed for other variables. Sequencing data for all detected taxa were linked to survey responses using a unique identification. Averages and SD of the number of species and genera detected in both IBS and control groups were calculated. Geometric mean pairwise ratios were used to calculate size factors to adjust for varying sequencing depths. Counts of taxa with less than 30 non-zero counts were amalgamated into a single column labelled 'Others' ${ }^{30}$ Bayesian inference with a Dirichlet prior was used to replace zero values, and a multiplicative modification of the non-zero values to maintain proportions. ${ }^{31}$ A CLR transformation was then applied to account for the compositional nature of the data. All subsequent analyses were performed on these transformed data.

Wilcoxon rank-sum tests were performed to select taxa that show significant location shifts between IBS and control groups; $p$ values were adjusted for multiple testing by controlling the false discovery rate (presented as $q$ values). All significantly different taxa were selected for multivariable testing. Mixed-effects models were fit, using laboratory (sequencing run) and geographical (state, region) variables as random intercepts. These variables explained less than $1 \%$ of variation in the data (adjusted intraclass correlation $<0.01$ ). Hence, a fixed-effects multivariable logistic regression model was fit starting with all predictors. Due to the exploratory nature of this analysis, all taxa variables were retained in the model. Age, sex, race, antibiotic use in the preceding year, and family history of GI disorders were included in the model as potential confounders. Second-order and third-order interactions were checked for all confounder variables using likelihood ratio tests. Model fit was assessed by plotting simulated residuals and conducting goodnessof-fit tests. ORs and CIs were generated from the models. ORs represent changes in odds of disease associated with changes in taxa on the CLR scale. To aid interpretation, ORs were back-transformed so that they reflect changes in odds of disease associated with intertaxa ratios; that is, these 'pairwise' ORs represent the change in odds of IBS when the ratio of one species to another increases by unit amount. These were visualised as a heatmap for all possible pairs of species that were statistically significant in the multivariable model. A p value (or $q$ value) of less than 0.05 was considered significant. The same procedure was applied to a subgroup to explore IBS among individuals reporting antibiotic use. All analyses were done in the $\mathrm{R}$ programming language. ${ }^{32}$

\section{RESULTS}

Of 28878 surveys distributed, 6386 responses (22.1\%) were received. A third of these responders $(n=2116)$ reported a diagnosis of IBS at the time of their most recent stool sample. Of these, 1692 reported no other comorbidities, and these individuals comprise the IBS group. Findings from this group were compared with a control group of 1124 individuals reporting no health conditions (figure 1).

Participant characteristics are described in table 1. Individuals in the IBS group were predominantly female. While age and region of the USA were largely comparable between the groups, a slightly higher proportion of controls resided in Western USA. The majority of the cohort identified as Caucasian, with a large portion not responding to race and ethnicity questions. More participants in the IBS group reported having taken antibiotics in the previous year than in the control group, and more reported a family history of gut disorders.

Taxonomic annotation was performed as described in the Methods section. A total of 15 phyla, 364 genera, and 807 species were detected across all samples. Of these, 6 phyla, 277 genera, and 635 species had low counts $(<30)$ and were amalgamated into a single column labelled 'Others' for each taxa-level analysis, respectively. On average, $4.59(\mathrm{SD}=0.93)$ phyla, $22.00(\mathrm{SD}=6.24)$ genera, and $28.40(\mathrm{SD}=9.17)$ species were detected in the IBS group vs $4.80(\mathrm{SD}=0.90)$ phyla, $21.70(\mathrm{SD}=5.90)$ genera, and $28.20 \quad(\mathrm{SD}=9.26)$ species in the control group. Among those in the IBS group, the most commonly detected phylum was Bacteroidetes at $54.52 \%$, followed by Firmicutes $(34.85 \%)$, Actinobacteria $(3.73 \%)$, Verrucomicrobia $(3.18 \%)$, and Proteobacteria $(3.07 \%)$. In the control group, the most commonly detected phylum was also Bacteroidetes at $52.44 \%$, followed by Firmicutes $(37.17 \%)$, Actinobacteria $(3.99 \%)$, Proteobacteria $(2.99 \%)$, and Verrucomicrobia $(2.87 \%)$.

To facilitate interpretation, CLRs of the abundance of select species in each group are shown in a boxplot in figure 2. Species-level results of univariate testing of differences in abundance are presented in online supplementary table A1. After amalgamation, 154 species, 70 genera, and 4 phyla were found significantly different across the IBS and control groups (Wilcoxon rank-sum tests shown in online supplementary tables A1, A3 and A5).

All taxa significant at $\mathrm{q}<0.05$ were included in separate logistic regression models for species and genera, while phyla significant at $\mathrm{q}<0.20$ were included in another model. Results of selected multivariable analyses are 


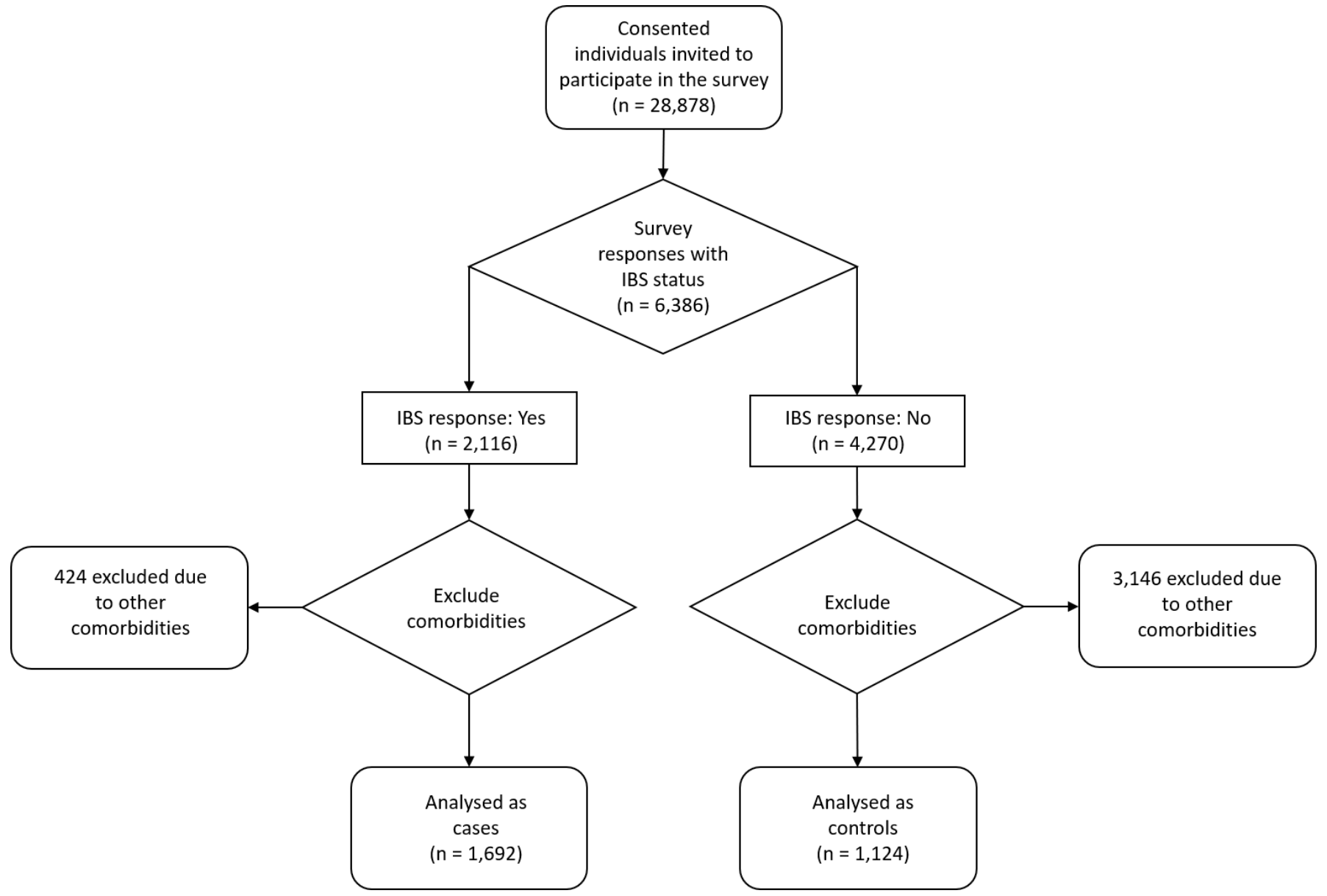

Figure 1 Recruitment flow chart. The flow chart describes the study selection and allocation of individuals into case and control groups. IBS, irritable bowel syndrome.

shown in table 2 (full list shown in online supplementary tables A2, A4 and A6). Three phyla, 19 species, and 15 genera remained significant on adjustment for age, sex, use of antibiotics in the preceding year and family history of GI disorders. For most of these species and genera, a unit increase in the CLR was associated with an increase in the odds of having IBS. The species-level heatmap of pairwise ORs illustrates the complexity of compositional differences in the microbiome (figure 3). Genus-level and phylum-level results are presented in online supplementary figures A1 and A2.

A family history of IBS, defined as having a biological parent or sibling diagnosed with IBS, was associated with a 5.15-fold increase in the odds of having IBS (95\% CI 3.80 to 6.98) in the species-level analysis; similar results were seen at the genus and phylum level. Among other demographic factors, sex and age were not independently significantly associated with IBS at the species level; however, an age-sex interaction was significant $\left(\chi^{2}=49.25, \mathrm{df}=34, \mathrm{p}=0.04\right)$. That is, the odds of IBS drop with increasing age for men as compared with women in similar age groups. The only behavioural factor in the model, antibiotic usage in the preceding year, was strongly and independently associated with IBS. Use of antibiotics from most classes was associated with an increase in odds of IBS. Quinolones showed the largest significant effect size at the species and genus level, that is, OR 2.06 (95\% CI 1.37 to 3.10) and 1.94 (95\% CI 1.27 to 2.96$)$, respectively.

Among participants reporting antibiotic use in the preceding year, the majority also reported a diagnosis of IBS $(73.3 \%$ ) (online supplementary table A7). The IBS group reported lower use of penicillins and higher use of quinolones compared with those reporting no health conditions. Further, those reporting a diagnosis of IBS reported family history of gut conditions more frequently than their non-IBS counterparts. Species-level pairwise ORs in this subgroup differ from the overall cohort (online supplementary figure A3; genus-level online supplementary figure A4). For example, the relationship between Streptococcus agalactiae and the other significant species was stronger in the antibiotics subgroup than in the overall cohort; an increase in $S$. agalactiae at the expense of all other significant species was associated with higher increased risk of IBS in the antibiotics subgroup than in the full cohort. 
Table 1 Participant characteristics and distribution in the control and IBS groups $(n=2816)$

\begin{tabular}{|c|c|c|c|}
\hline & Control $(n=1124)$ & IBS (n=1692) & $P$ value \\
\hline Age, $n$, median (25th-75th percentile) & $401124(34-50)$ & $421692(34-53)$ & 0.01 \\
\hline \multicolumn{4}{|l|}{ Gender, n (\%) } \\
\hline Female & $724(64.41)$ & $1290(76.24)$ & $<0.01$ \\
\hline Male & $400(35.59)$ & $402(23.76)$ & $<0.01$ \\
\hline \multicolumn{4}{|l|}{ Region of the USA, $n(\%)$} \\
\hline Midwest & $183(16.28)$ & $289(17.08)$ & 0.61 \\
\hline Northeast & $202(17.97)$ & $342(20.21)$ & 0.15 \\
\hline South & $282(25.09)$ & $478(28.25)$ & 0.07 \\
\hline West & $457(40.66)$ & $583(34.46)$ & $<0.01$ \\
\hline \multicolumn{4}{|l|}{ Race, n (\%) } \\
\hline Caucasian/European/American & $606(53.91)$ & $1011(59.75)$ & $<0.01$ \\
\hline African-American & $10(0.89)$ & $25(1.48)$ & 0.22 \\
\hline Asian/Oceanian/Pacific Islander/Hawaiian & $52(4.63)$ & $33(1.95)$ & $<0.01$ \\
\hline Latin American/Hispanic & $38(3.38)$ & $34(2.01)$ & 0.03 \\
\hline Mixed & $35(3.11)$ & $33(1.95)$ & 0.06 \\
\hline Unknown & $383(34.08)$ & $556(32.86)$ & 0.52 \\
\hline Antibiotics in the preceding year, $\mathrm{n}(\%)$ & 349 (31.05) & $1283(75.83)$ & $<0.01$ \\
\hline Penicillins & $160(45.85)$ & $416(32.42)$ & $<0.01$ \\
\hline Tetracyclines & $45(12.89)$ & $184(14.34)$ & 0.54 \\
\hline Cephalosporins & $23(6.59)$ & $110(8.57)$ & 0.27 \\
\hline Quinolones & $37(10.60)$ & $211(16.45)$ & $<0.01$ \\
\hline Lincomycins & $8(2.29)$ & $59(4.60)$ & 0.07 \\
\hline Macrolides & $62(17.77)$ & $211(16.45)$ & 0.61 \\
\hline Sulfonamides & $11(3.15)$ & $67(5.22)$ & 0.14 \\
\hline Glycopeptides & $1(0.29)$ & $19(1.48)$ & $<0.01$ \\
\hline Aminoglycosides & $2(0.57)$ & $6(0.47)$ & 0.28 \\
\hline Family history of gut disorders, n (\%) & 193 (17.17) & 760 (44.92) & $<0.01$ \\
\hline IBS & $61(31.61)$ & $431(56.71)$ & $<0.01$ \\
\hline Ulcerative colitis & $26(13.47)$ & $58(7.63)$ & 0.01 \\
\hline Crohn's disease & $29(17.79)$ & $58(7.63)$ & $<0.01$ \\
\hline Diverticulitis & $77(39.90)$ & $213(28.03)$ & $<0.01$ \\
\hline
\end{tabular}

Antibiotics in the preceding year and family history of gut disorders contain groups that are not mutually exclusive. Bolded values in table denote aggregate counts.

IBS, irritable bowel syndrome.

\section{DISCUSSION}

Although IBS is a highly prevalent and widely studied functional GI disorder, fully defining its pathophysiology, aetiology, and clinical characteristics has historically proven difficult. The disorder presents a challenge to clinicians as well as patients, as it commonly presents with heterogeneous and non-specific symptoms that significantly affect quality of life. ${ }^{2}$ Previous studies have shown an alteration of gut microbiota composition, temporal stability, and diversity in patients with IBS. ${ }^{33} 34$ In the current study, we characterise a large cohort reporting a diagnosis of IBS in terms of demographics, symptoms, risk factors, and microbiome composition. We performed an exploratory analysis to investigate microbiome differences in
IBS compared with a control group. To account for the multifactorial nature of IBS, we performed a multivariable analysis to control for potential confounding factors, including age, sex, race, antibiotic usage, and family history. These factors are known to influence microbiome composition $^{12}$ and are also known risk factors for IBS. ${ }^{35}$

Using this approach, we identified several interesting organisms associated with significant ORs in cases versus controls. First, an increased abundance of the genus Streptococcus, as well as the species S. agalactiae, was associated with a significantly increased odds of IBS. Streptococcus has been previously associated with Irritable Bowel Syndrome with Diarrhea (IBS-D) ${ }^{33}$ and inflammatory bowel disease, ${ }^{34}$ perhaps suggesting a general association 


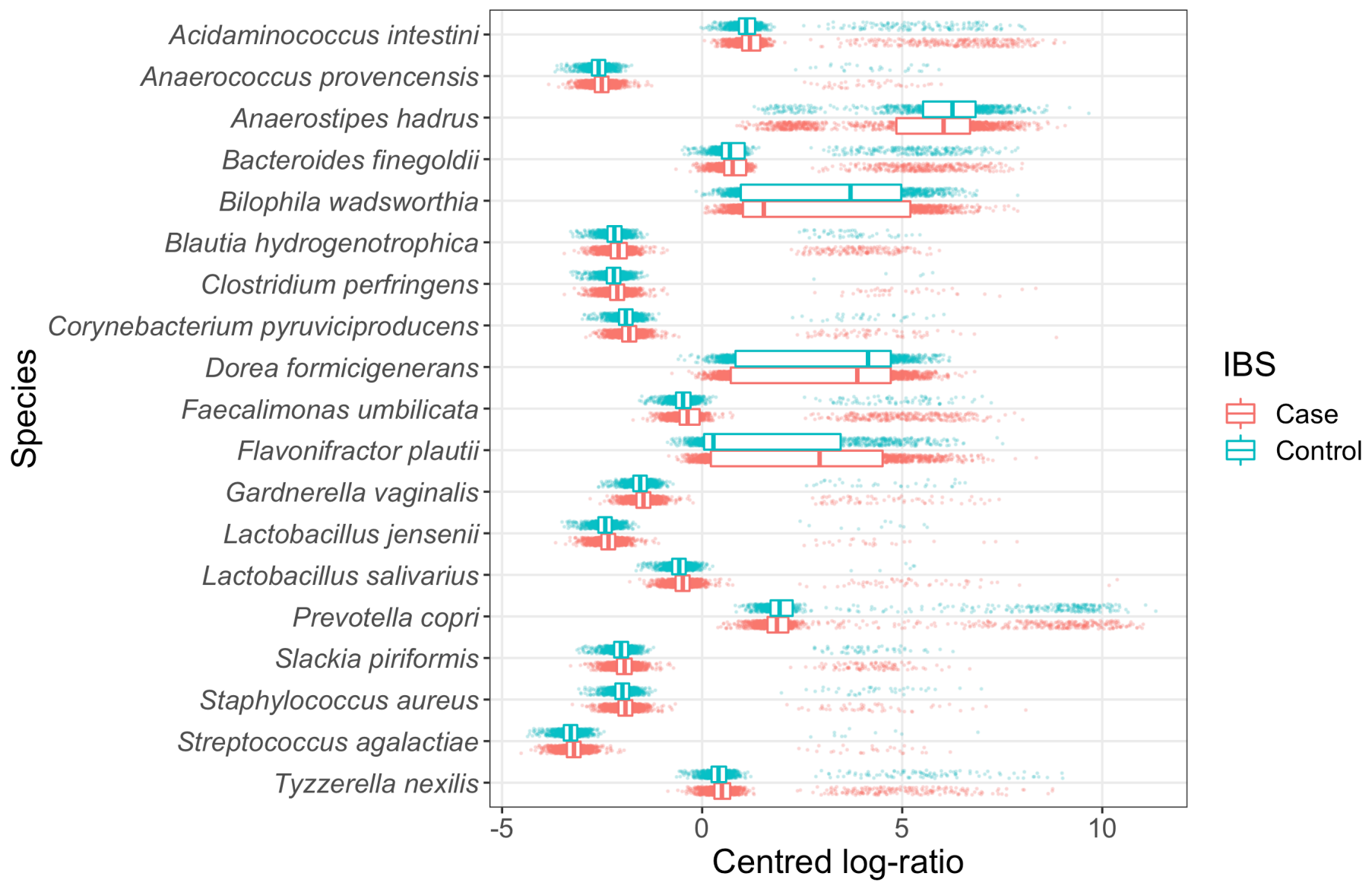

Figure 2 Transformed abundances of selected species. Centred log ratio (CLR) of statistically significantly different species among irritable bowel syndrome (IBS) and control groups. CLR is calculated by taking the log of the ratio between the number of reads of a given species and the geometric mean of all species for a given sample. Points in the plot are the CLR abundance for each participant sample. Superimposed boxplots show the median (central line) and the 25th and 75th percentiles (left and right edges of the box, respectively) of the distribution of CLR abundances across all individuals in the data set.

with bowel dysfunction. At the species level, S. agalactiae (group B strep), a commensal with the potential to cause disease, has not been associated with IBS previously; additional species-level studies are needed to explore such relationships.

We also identified Gardnerella vaginalis as significantly increasing the odds of IBS by $16 \%$. Further investigation into the data set revealed that $G$. vaginalis was detected in 99 samples, 97 of which were from women, suggesting that the detection of $G$. vaginalis in these stool samples may represent translocation or contamination from the vagina before or during sampling, respectively. Interestingly, of these 97 samples, $68 \%$ were IBS cases. The presence of $G$. vaginalis in the vagina is usually indicative of bacterial vaginosis, a similarly poorly defined disease characterised by a disturbance of the vaginal microbiome. ${ }^{36} 37$ Possible co-occurrence of bacterial vaginosis and IBS may reflect an overall loss of microbial diversity, perhaps from overexposure to antibiotics or consuming a suboptimal diet, and warrants further study.

Interestingly, the genus Lactobacillus, and specifically L. jensenii and L. salivarius, was associated with increased odds of IBS. Many species of the GI tract belong to the genus Lactobacillus, some of which are considered probiotic. ${ }^{38}$ Previous studies have reported conflicting associations with IBS; for example, in a recent systematic review of 24 case-control studies, the family Lactobacillaceae was increased in IBS versus controls, ${ }^{20}$ and in a separate meta-analysis the genus Lactobacillus was decreased in IBS cases. ${ }^{17}$ In an in vitro study, specific strains of $L$. salivarius induced proinflammatory cytokine production from macrophages, while other strains induced antiinflammatory cytokines, providing an intriguing mechanistic explanation and suggesting differences at the strain level that have not been captured in microbiome association studies. ${ }^{39}$ We also cannot rule out that we are detecting Lactobacillus in the stool from oral probiotic use, as IBS sufferers may be more likely to consume probiotics. $^{38}$

Other organisms of interest found to be associated with increased odds of IBS in this cohort include the genus Veillonella as well as the species Prevotella copri. Veillonella has been previously reported to be increased in IBS compared with controls. ${ }^{1540}$ While the genus Prevotella is abundant in the human microbiome and considered a commensal, there is ample evidence that many species may promote inflammation ${ }^{14}{ }^{41}$; specifically, an increase in P. copri was correlated with new-onset rheumatoid 


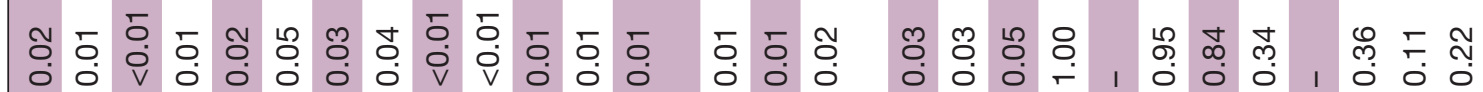

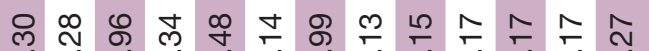

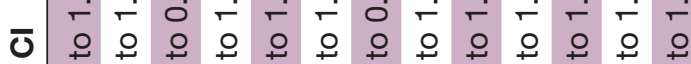

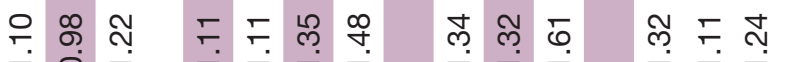

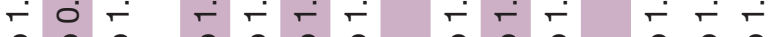

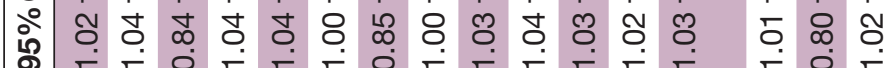
와 우 웅ㅇㅇㅇㅛ

웅요

+2 웅

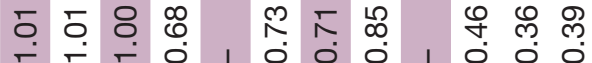

뜬

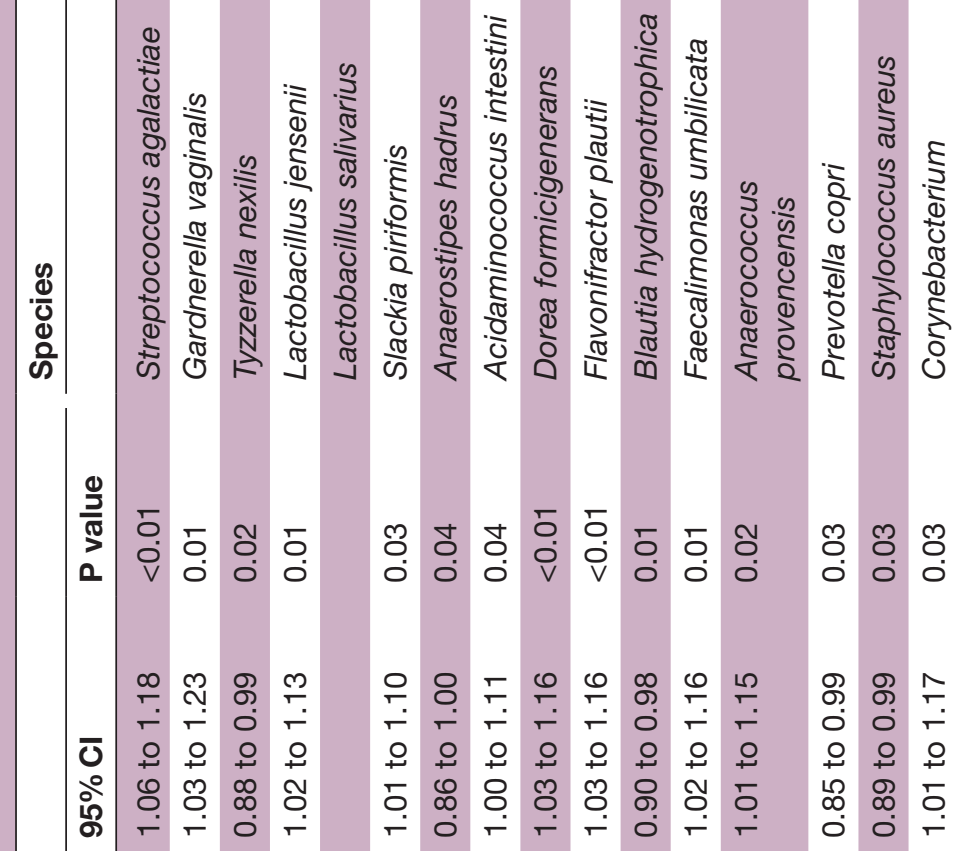

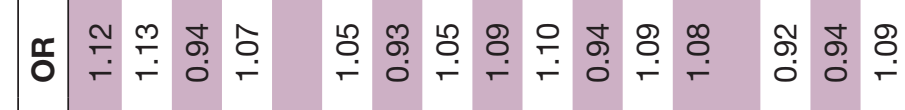

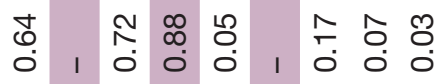
중

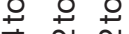

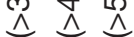

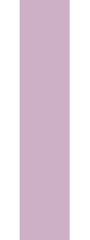




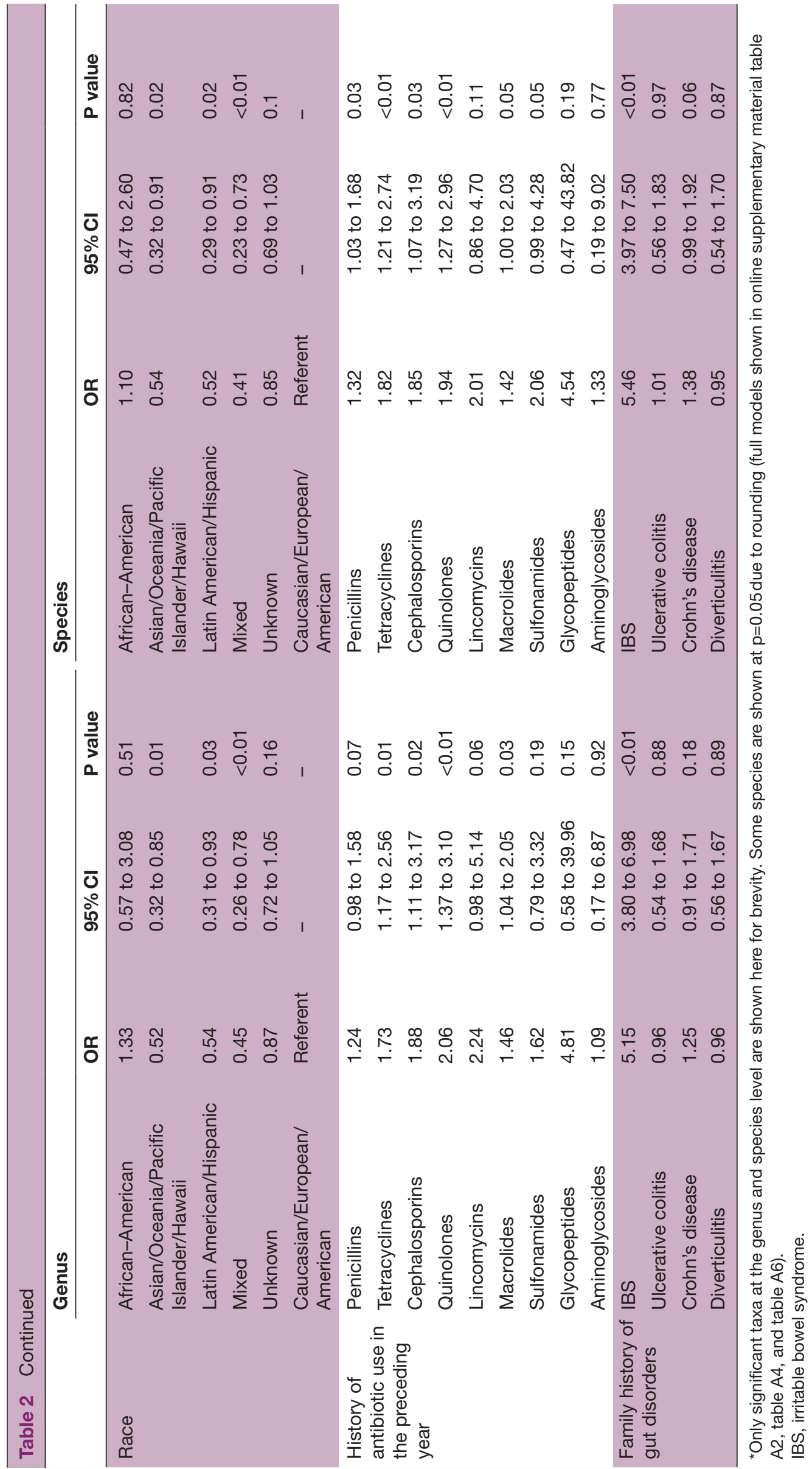




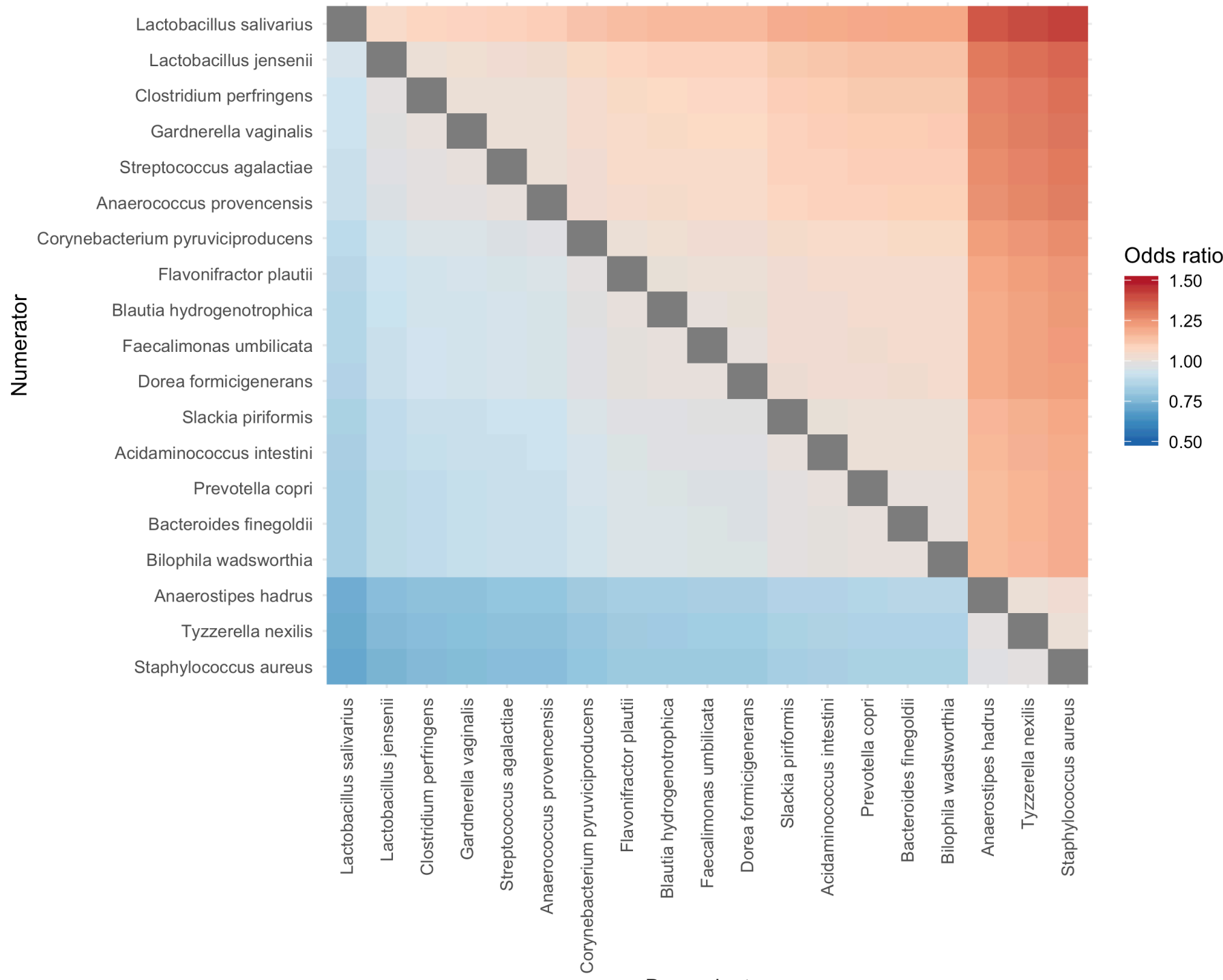

Denominator

Figure 3 Pairwise ORs of gut microbiome species for IBS versus control individuals. The colour of each tile in the heatmap represents the OR associated with a unit change in the ratio between two species in the microbiome. The numerators of these ratios are on the $y$-axis, while the denominators are on the $\mathrm{x}$-axis. IBS, irritable bowel syndrome.

arthritis in a relatively large cohort of patients, and was shown to intensify colitis phenotype in a mouse model. ${ }^{42}$ Although IBS is not traditionally associated with inflammation, recent studies suggest low-grade inflammation as contributory. ${ }^{43}$

We identified Faecalibacterium as protective against IBS in this cohort. Previous studies have shown that a member of this genus, Faecalibacterium prausnitzii, has an important anti-inflammatory role and is frequently observed to be decreased in inflammation-related gut disorders. ${ }^{21}{ }^{44} \mathrm{We}$ also found that the genera Coprococcus and Anaerostipes were associated with significantly decreased odds of IBS, along with a few other genera and species of which there has not been much information previously reported. The large sample size of this study offers an opportunity for multivariable analysis at several taxonomic levels with IBS as the outcome. Further, this allows for examination of the effects of individual taxa on the odds of IBS while adjusting for other taxa, substantially adding to existing knowledge of this disorder.

Surprisingly, we identified Staphylococcus aureus as associated with decreased odds of IBS. An opportunistic pathogen, S. aureus has also been implicated as one of the initial infectious agents that can trigger postinfectious IBS (PI-IBS). ${ }^{18}$ We hypothesise that the inverse association with IBS found in the current study may be due to the statistically significant increased use of antibiotics among patients with IBS $(75.83 \%)$ compared with those without IBS $(31.05 \%)$, which could dramatically decrease S. aureus in that group. Clostridium perfringens, an enteric pathogen, was associated with an increased risk of IBS and may suggest a role in the pathogenesis of PI-IBS. Indeed, studies report that $6 \%-17 \%$ of IBS cases are attributed to GI infections. ${ }^{45}$

When species abundance was analysed in a pairwise fashion, we were able to identify pairwise ratios of 
organisms that were significantly associated with the odds of having IBS. This analysis highlights how compositional variations in the microbiome relate to disease outcome and reflects the reality that each species does not exist alone in the gut environment. Interestingly, increased $L$. salivarius at the expense of $S$. aureus showed the largest association on the odds of IBS. This may reflect increased probiotic and antibiotic use in the IBS group, or a potential ecological interdependence that has not been explored. The pairwise ORs for Anaerostipes hadrus, Tyzzerella nexilis and S. aureus with respect to the other species are interesting, as the relationships suggest interactions which may lead to or result from dysbiosis. Several recent studies and reviews underscore the often contradictory microbial changes among patients with IBS, ${ }^{24647}$ offering support for an ecological shift in the gut microbiome as a critical component of IBS pathology. The species-level pairwise analysis generates hypotheses for future mechanistic studies to explore potential interactions between species in the gut.

We also confirmed previous observations regarding non-microbial risk factors for IBS. Over $48 \%$ of individuals in the IBS group reported having a first-degree relative with a diagnosed GI condition, compared with $18 \%$ in the control group. IBS has been shown to cluster within families, ${ }^{8}$ indicating a potential genetic or environmental component. Confirming previous reports, ${ }^{12}$ we also found that over $75 \%$ of individuals in the IBS group reported using antibiotics in the last year, compared with only $30 \%$ in the control group. The use of quinolones specifically was strongly associated with the IBS group; previous studies on ciprofloxacin have shown a widespread effect on the gut microbiome. ${ }^{48}$

About $10 \%$ of patients with acute infectious enteritis will go on to develop PI-IBS, ${ }^{49}$ and the risk of PI-IBS is increased in those who used antibiotics during enteritis. ${ }^{50}$ The association of antibiotic use with IBS in this cohort may be a reflection of an underlying high prevalence of PI-IBS; more likely it is evidence of multiple unsuccessful courses of antibiotics aiming to empirically treat GI symptoms. These exposures contribute to the aetiology of IBS by disrupting the host-microbial balance.

Pairwise species abundance analysis within the subgroup of participants reporting antibiotic use in the last year revealed associations and microbiome composition distinct from the overall cohort. This suggests that while antibiotics undoubtedly disrupt the structure of the microbiome in the entire subgroup, there may be specific microbial patterns that mediate susceptibility or resiliency to IBS. It should be noted that the cross-sectional nature of the study precludes temporal associations between antibiotic use, previous infection, and diagnosis of IBS; however, these results warrant further study.

A notable strength of this study is the uniquely large sample size, which allowed us to adjust for relevant variables that are important in a complex disorder such as IBS. Moreover, the ability to distinguish between species is relevant for uncovering novel associations that would otherwise be buried at the genus level. When investigating microbiome associations, this is a relatively novel approach that lays the groundwork for future predictive modelling. In addition, it is often challenging to pool results across studies as varied sample collection, sequencing, and analysis procedures can lead to differences in reported microbiome composition, ${ }^{19}$ further demonstrating the need for studies on large cohorts such as this. As knowledge of the microbiome in IBS and other complex diseases increases, analyses like this may lead to useful discoveries to assist in diagnosis, treatment, and prevention.

\section{Limitations and future research}

There are several limitations to this work. First, this study reports findings from a large convenience sample and may not be a representative cohort. However, the sample size of this study reveals findings that might otherwise be obscured in a smaller cohort. The self-reported nature of this study is a limitation, as it was not feasible in a cohort of this size to perform reviews of medical records. The cohort consists of individuals residing in the USA, and it is unclear how the microbiome differences reported here can be generalised worldwide. As diet and environment play major roles in shaping the gut microbiome, future studies must include populations from other parts of the world. Last, the annotation pipeline is optimised for high precision at the expense of some sensitivity.

This cross-sectional study was not designed to capture temporal associations and, similar to other studies investigating the microbiome in IBS to date, is associative in nature and no assumptions about causality can be made. However, the comprehensive demographic, clinical, and phenotypic data we collected and the large population allowed us to build exploratory models that can inform future studies. Both longitudinal studies of patients with IBS as well as prospective epidemiological studies to assess risk are needed to more fully understand the role of the microbiome in IBS and other chronic gut conditions.

Much of IBS treatment is varied and often not effective long term. ${ }^{9}{ }^{10}$ For example, motility agents may work upstream from the microbial influence on IBS. Nonabsorbable antibiotics such as rifaximin are often tried empirically and can be effective, but tend to mostly work on those with small intestine bacterial overgrowth. ${ }^{51}$ Probiotics have been shown to improve IBS symptoms in some cases, ${ }^{2}$ but the optimal combination and dose are yet to be determined. Dietary restriction with low fermentable oligo-, di-, mono-saccharides and polyols (FODMAPs) is also used with mixed success. ${ }^{52}$ Therefore, it is plausible that certain microbiome compositions may indicate improved candidacy for rifaximin or specific dietary strategies, ${ }^{53}$ which would necessitate more personalised IBS therapies in this very heterogeneous disease. Microbiome testing and monitoring may be beneficial adjuncts to conventional care pathways. Future studies should examine microbial-based monitoring and therapeutic interventions as higher-resolution tools become 
available; indeed, they are poised to significantly improve clinical outcomes in this population.

\section{CONCLUSION}

More than $11 \%$ of the global population suffers from IBS, and research continues to support a dynamic mix of risk factors contributing to IBS pathophysiology. Multifaceted conditions such as IBS pose a challenge for diagnosis and treatment as the circumstances for disease occurrence vary based on internal and external conditions. This study leverages a large cohort to identify gut microbiome differences among individuals reporting an IBS diagnosis, and identifies 3 phyla, 15 genera, and 19 species as significantly associated with IBS after adjustment for confounding factors. Further, the results highlight interesting relationships among commensal and pathogenic species, and suggest an important role for dysbiosis in disease pathophysiology. Due to its heterogeneous nature, there may not be an IBS-specific gut microbiome signature, but rather a cumulative alteration in the gut microbiome leading to dysbiosis and increased risk of chronic gastroenterological conditions. Largescale, ecological-focused studies are needed to better understand the conditions for IBS pathogenesis, and may provide key insights to improve clinical guidelines for the diagnosis, monitoring and treatment of IBS.

Twitter Michael C Hoaglin @drmike

Acknowledgements The authors gratefully acknowledge support from Luis Leon, Felipe Melis and Raul Arias for the bioinformatics pipeline used in the study. Further, the authors thank Susan Zneimer and Eduardo Morales for thoughtful comments and review.

Contributors SG, DEA, MH, and $\mathrm{CP}$ were involved in conceptualising the study. LNC, SG, DEA, and MCH supervised the work. Survey design and data collection were completed by $\mathrm{MCH}$ and $\mathrm{MH}$, with support from CG. Bioinformatics and statistical analyses were led by NI, with key contributions from RP, IV, and MH. MA, LNC, and NI guided the data interpretation along with CG, MH, RP, DEA, and MCH. LNC and MA led the efforts to write and edit the manuscript. NI, CP, MH, and MCH wrote portions of the original draft, and all authors reviewed the final draft for edits prior to submission.

Funding uBiome funded the study design, collection, analysis and interpretation of the data; writing of the paper; and decision to submit for publication. Manuscript publication was funded by Psomagen.

Competing interests All of the authors were employees or contractors of uBiome at the time of this work and received compensation. Some authors have issued and/or pending patents in relation to this work: patent US 9,703,929 issued, patent US 10,242,160 issued, patent US 10,287,639 issued, patent US 10,294,532 issued, patent US 10,331,857 issued, patent US 10,360,346 issued, patent US 2017/0270268 pending, patent US 2018/0137239 pending, and patent US $2019 / 0050534$ pending. All authors have a patent pending in relation to this work: US provisional application No 62/968,251, Gut microbiome composition and risk factors in a large cross-sectional IBS cohort.

Patient consent for publication Not required.

Ethics approval The study was approved by Asentral IRB (\#2016-440).

Provenance and peer review Not commissioned; externally peer reviewed.

Data availability statement Data are available upon reasonable request. Please contact the corresponding author.

Open access This is an open access article distributed in accordance with the Creative Commons Attribution Non Commercial (CC BY-NC 4.0) license, which permits others to distribute, remix, adapt, build upon this work non-commercially, and license their derivative works on different terms, provided the original work is properly cited, appropriate credit is given, any changes made indicated, and the use is non-commercial. See: http://creativecommons.org/licenses/by-nc/4.0/.

\section{ORCID iDs}

Lauren N Carroll http://orcid.org/0000-0003-0739-5861

Maureen Hitschfeld http://orcid.org/0000-0001-7322-875X

Michael C Hoaglin http://orcid.org/0000-0003-4643-8617

\section{REFERENCES}

1 Frank DN, Pace NR. Gastrointestinal microbiology enters the metagenomics era. Curr Opin Gastroenterol 2008;24:4-10.

2 Simrén M, Barbara G, Flint HJ, et al. Intestinal microbiota in functional bowel disorders: a Rome Foundation report. Gut 2013:62:159-76.

3 Moayyedi P, Mearin F, Azpiroz F, et al. Irritable bowel syndrome diagnosis and management: a simplified algorithm for clinical practice. United European Gastroenterol J 2017;5:773-88.

4 Lacy BE, Patel NK. Rome criteria and a diagnostic approach to irritable bowel syndrome. J Clin Med 2017;6. doi:10.3390/ jcm6110099. [Epub ahead of print: 26 Oct 2017].

5 American Gastroenterological Association. Ibs in America, 2015. Available: https://www.gastro.org/guidelines/ibd-and-boweldisorders\%20on\%20September\%201 [Accessed 1 Sep 2018].

6 Lacy B, Ayyagari R, Guerin A, et al. Factors associated with more frequent diagnostic tests and procedures in patients with irritable bowel syndrome. Therap Adv Gastroenterol 2019;12:175628481881832.

7 Hungin APS, Molloy-Bland M, Claes R, et al. Systematic review: the perceptions, diagnosis and management of irritable bowel syndrome in primary care--a Rome Foundation working team report. Aliment Pharmacol Ther 2014;40:1133-45.

8 Canavan C, West J, Card T. The epidemiology of irritable bowel syndrome. Clin Epidemiol 2014;6:71-80.

9 Lacy BE, Weiser K, De Lee R. The treatment of irritable bowel syndrome. Therap Adv Gastroenterol 2009;2:221-38.

10 Harris LA, Baffy N. Modulation of the gut microbiota: a focus on treatments for irritable bowel syndrome. Postgrad Med 2017;129:872-88.

11 Menees S, Chey W. The gut microbiome and irritable bowel syndrome. F1000Res 2018;7. doi:10.12688/f1000research.14592.1. [Epub ahead of print: 09 Jul 2018].

12 Collins SM. A role for the gut microbiota in IBS. Nat Rev Gastroenterol Hepatol 2014;11:497-505.

13 Halvorson HA, Schlett CD, Riddle MS. Postinfectious irritable bowel syndrome--a meta-analysis. Am J Gastroenterol 2006;101:1894-9.

14 Tap J, Derrien M, Törnblom H, et al. Identification of an intestinal microbiota signature associated with severity of irritable bowel syndrome. Gastroenterology 2017;152:111-23.

15 Tana C, Umesaki Y, Imaoka A, et al. Altered profiles of intestinal microbiota and organic acids may be the origin of symptoms in irritable bowel syndrome. Neurogastroenterol Motil 2010;22:512-9.

16 Putignani L, Del Chierico F, Vernocchi P, et al. Gut microbiota dysbiosis as risk and premorbid factors of IBD and IBS along the Childhood-Adulthood transition. Inflamm Bowel Dis 2016;22:487-504.

17 Liu H-N, Wu H, Chen Y-Z, et al. Altered molecular signature of intestinal microbiota in irritable bowel syndrome patients compared with healthy controls: a systematic review and meta-analysis. Dig Liver Dis 2017:49:331-7.

18 Bennet SMP, Öhman L, Simrén M. Gut microbiota as potential orchestrators of irritable bowel syndrome. Gut Liver 2015;9:318-31.

19 Salonen A, de Vos WM, Palva A. Gastrointestinal microbiota in irritable bowel syndrome: present state and perspectives. Microbiology 2010;156:3205-15.

20 Pittayanon R, Lau JT, Yuan Y, et al. Gut microbiota in patients with irritable bowel syndrome-a systematic review. Gastroenterology 2019:157:97-108.

21 Rajilić-Stojanović M, Biagi E, Heilig HGHJ, et al. Global and deep molecular analysis of microbiota signatures in fecal samples from patients with irritable bowel syndrome. Gastroenterology 2011;141:1792-801.

22 von Elm E, Altman DG, Egger M, et al. The strengthening the reporting of observational studies in epidemiology (STROBE) statement: guidelines for reporting observational studies. J Clin Epidemiol 2008:61:344-9.

23 Hummel W, Kula M-R. Simple method for small-scale disruption of bacteria and yeasts. J Microbiol Methods 1989;9:201-9. 
24 Cady NC, Stelick S, Batt CA. Nucleic acid purification using microfabricated silicon structures. Biosens Bioelectron 2003;19:59-66.

25 Caporaso JG, Lauber CL, Walters WA, et al. Global patterns of $16 \mathrm{~S}$ rRNA diversity at a depth of millions of sequences per sample. Proc Natl Acad Sci U S A 2011;108 Suppl 1:4516-22.

26 Minalla AR, Dubrow R, Bousse L. Feasibility of high-resolution oligonucleotide separation on a microchip. Proceedings of SPIE The International Society for Optical Engineering 2001:4560.

27 Mahé F, Rognes T, Quince C, et al. Swarm: robust and fast clustering method for amplicon-based studies. PeerJ 2014;2:e593.

28 Rognes T, Flouri T, Nichols B, et al. VSEARCH: a versatile open source tool for metagenomics. PeerJ 2016;4:e2584.

29 Pruesse E, Quast C, Knittel K, et al. Silva: a comprehensive online resource for quality checked and aligned ribosomal RNA sequence data compatible with ARB. Nucleic Acids Res 2007;35:7188-96.

30 Aitchison J. The statistical analysis of compositional data. Journal of the Royal Statistical Society: Series B 1982;44:139-60.

31 Martín-Fernández J-A, Hron K, Templ M, et al. Bayesianmultiplicative treatment of count zeros in compositional data sets. Stat Modelling 2015;15:134-58.

32 R Core Team. R: a language and environment for statistical computing. Vienna, Austria: R Foundation for Statistical Computing, 2018. https://www.r-project.org/

33 Kassinen A, Krogius-Kurikka L, Mäkivuokko H, et al. The fecal microbiota of irritable bowel syndrome patients differs significantly from that of healthy subjects. Gastroenterology 2007;133:24-33.

34 Lo Presti A, Zorzi F, Del Chierico F, et al. Fecal and mucosal microbiota profiling in irritable bowel syndrome and inflammatory bowel disease. Front Microbiol 2019;10:1655.

35 Chey WD, Kurlander J, Eswaran S. Irritable bowel syndrome: a clinical review. JAMA 2015;313:949-58.

36 Schwebke JR, Muzny CA, Josey WE. Role of Gardnerella vaginalis in the pathogenesis of bacterial vaginosis: a conceptual model. J Infect Dis 2014;210:338-43.

37 Shipitsyna E, Roos A, Datcu R, et al. Composition of the vaginal microbiota in women of reproductive age--sensitive and specific molecular diagnosis of bacterial vaginosis is possible? PLoS One 2013;8:e60670.

38 Hosseini A, Nikfar S, Abdollahi M. Probiotics use to treat irritable bowel syndrome. Expert Opin Biol Ther 2012;12:1323-34.

39 Drago L, Nicola L, lemoli E, et al. Strain-Dependent release of cytokines modulated by Lactobacillus salivarius human isolates in an in vitro model. BMC Res Notes 2010;3:44.
40 Saulnier DM, Riehle K, Mistretta T-A, et al. Gastrointestinal microbiome signatures of pediatric patients with irritable bowel syndrome. Gastroenterology 2011;141:1782-91.

41 Larsen JM. The immune response to Prevotella bacteria in chronic inflammatory disease. Immunology 2017;151:363-74.

42 Scher JU, Sczesnak A, Longman RS, et al. Expansion of intestinal Prevotella copri correlates with enhanced susceptibility to arthritis. Elife 2013;2:e01202.

$43 \mathrm{Ng} \mathrm{QX}$, Soh AYS, Loke W, et al. The role of inflammation in irritable bowel syndrome (IBS). J Inflamm Res 2018;11:345-9.

44 Ferreira-Halder CV. Faria AV de S, Andrade SS. action and function of Faecalibacterium prausnitzii in health and disease. Best Pract Res Clin Gastroenterol 2017;31:643-8.

45 Sadeghi A, Biglari M, Nasseri Moghaddam S. Post-Infectious irritable bowel syndrome: a narrative review. Middle East J Dig Dis 2019;11:69-75.

46 Rodiño-Janeiro BK, Vicario M, Alonso-Cotoner $\mathrm{C}$, et al. A review of microbiota and irritable bowel syndrome: future in therapies. Adv Ther 2018;35:289-310.

47 Distrutti E, Monaldi L, Ricci P, et al. Gut microbiota role in irritable bowel syndrome: new therapeutic strategies. World J Gastroenterol 2016;22:2219-41.

48 lizumi T, Battaglia T, Ruiz V, et al. Gut microbiome and antibiotics. Arch Med Res 2017;48:727-34.

49 Barbara G, Grover M, Bercik P, et al. Rome Foundation working team report on Post-Infection irritable bowel syndrome. Gastroenterology 2019;156:46-58.

50 Klem F, Wadhwa A, Prokop LJ, et al. Prevalence, risk factors, and outcomes of irritable bowel syndrome after infectious enteritis: a systematic review and meta-analysis. Gastroenterology 2017;152:1042-54.

51 Pimentel M, Chow EJ, Lin HC. Eradication of small intestinal bacterial overgrowth reduces symptoms of irritable bowel syndrome. Am J Gastroenterol 2000;95:3503-6.

52 Nanayakkara WS, Skidmore PM, O'Brien L, et al. Efficacy of the low FODMAP diet for treating irritable bowel syndrome: the evidence to date. Clin Exp Gastroenterol 2016;9:131-42.

53 Chumpitazi BP, Cope JL, Hollister EB, et al. Randomised clinical trial: gut microbiome biomarkers are associated with clinical response to a low FODMAP diet in children with the irritable bowel syndrome. Aliment Pharmacol Ther 2015;42:418-27. 